\title{
Significance of Coronal Proset Magnetic Resonance Imaging to Detect Hidden Zone of the Mid-Zone Stenosis in the Lumbar Spine and Morphometric Analysis of the Mid-Zone Stenosis
}

\author{
Hyo-Sae Ahn, Whee Sung Son, Ji-Hoon Shin, Myun-Whan Ahn, Gun Woo Lee \\ Department of Orthopaedic Surgery, Yeungnam University Hospital, Yeungnam University College of Medicine, Daegu, Korea
}

\begin{abstract}
Study Design: Retrospective exploratory imaging study.
Purpose: To investigate the significance of the coronal magnetic resonance imaging (MRI) using Proset technique to detect the hidden zone in patients with mid-zone stenosis by comparing with conventional axial and sagittal MRI and to explore the morphologic characteristic patterns of the mid-zone stenosis.

Overview of Literature: Despite advancements in diagnostic modalities such as computed tomography and MRI, stenotic lesions under the pedicle and pars interarticularis, also called the mid-zone, are still difficult to definitely detect with the conventional axial and sagittal MRI due to its inherited anatomical peculiarity.

Methods: Of 180 patients scheduled to undergo selective nerve root block, 20 patients with mid-zone stenosis were analyzed using MRI. Characteristic group patterns were also explored morphologically by comparing MRI views of each group after verifying statistical differences between them. Hierarchical cluster analysis was performed to classify morphological characteristic groups based on three-dimensional radiologic grade for stenosis at all three zones.

Results: At the mid-zone, the stenosis of grade 2 or more was found in 14 cases in the coronal image, 13 cases in the sagittal image, and 9 cases in the axial image $(p<0.05)$. Especially, mid-zone stenosis was not detected in six of 20 cases at the axial images. At the entrance and exit-zone, coronal image was also associated with more accurate detection of hidden zone compared to other views such as axial and sagittal images. After repeated statistical verification, the morphological patterns of hidden zone were classified into 5 groups: 6 cases in group I; 1 case in group II; 4 cases in group III; 7 cases in group IV; and 2 cases in group V. Conclusions: Coronal MRI using the Proset technique more accurately detected hidden zone of the mid-zone stenosis compared to conventional axial and sagittal images.
\end{abstract}

Keywords: Spinal stenosis; Lumbar vertebrae; Magnetic resonance imaging; Proset; Mid zone

\section{Introduction}

In herniated intervertebral disc of the lumbar spine, the term "hidden zone" was first described by Macnab [1] in 1971 to convey the difficulty of detecting stenotic lesions at the mid-zone around the pedicle through conventional

\footnotetext{
Received Mar 3, 2016; Revised Apr 12, 2016; Accepted May 11, 2016

Corresponding author: Gun Woo Lee

Department of Orthopaedic Surgery, Yeungnam University Hospital, Yeungnam University College of Medicine,

170 Hyeonchung-ro, Nam-gu, Daegu 42415, Korea

Tel: +82-53-620-3644, Fax: +82-53-628-4020, E-mail: gwlee1871@gmail.com
} 
imaging studies. However, since the introduction of computed tomography (CT) and magnetic resonance imaging (MRI) in the 1980s, visualizing the hidden zone has become less problematic due to the acquisition of exquisite three-dimensional (3D) images with these new modalities. In addition, based on the advanced capabilities of these diagnostic modalities, several criteria for classifying compression lesions of the lumbar spine have been developed. Lee et al. [2] introduced a three-zone classification scheme, but pathologic lesions under the pars interarticularis at the mid-zone stenosis remain difficult to diagnose even with advanced modalities due to their anatomical peculiarity. In this region, the pathologic lesions are often obscured by the pedicle and the pars interarticularis during diagnostic imaging. Moreover, all diagnostic modalities are somewhat limited in their accuracy. From this standpoint, a hidden zone is still present in patients with the mid-zone stenosis.

Conventional diagnotic modalities, especially MRI, is also somewhat limited in the ability to detect the stenotic lesion of the mid-zone, but spine clinicians have not conducted any studies to find other method for identifying the hidden zone more accurately. In other words, there remains little choice for spine clinicians but to utilize conventional axial and sagittal MRI images, resulting from the lack of a referencing study. The authors thought that the coronal view of MRI might enable better definition of the mid-zone stenosis compared to the conventional axial and sagittal views, considering the anatomical location of the pedicle in the lumbar spine. Moreover, more advanced MRI technologies, such as Proset imaging, have the theoretically prominent features of water enhancement and lipid suppression, and can more sensitively detect pathological changes of the nerve root, such as swelling of nerve roots, thereby enabling more a precise evaluation of otherwise obscured lesions [3-6]. However, no radiological studies using coronal MRI views and the Proset technique have been done to compare the detection rate of the midzone stenosis and to reveal characteristic morphological patterns of mid-zone stenosis or any therapeutic guidelines based on these patterns.

In the current study, the authors aimed to evaluate the effcacy of the coronal MRI view, especially with Proset imaging, to detect mid-zone stenosis compared to the conventional axial and sagittal MRI views. In addition, this study explored the morphologic characteristic patterns of the mid-zone stenosis with 3D MRI views, based on the axial and sagittal as well as coronal MRI view, and to further suggest a basis for reasonable surgical decisions using these characteristic patterns.

To our knowledge, this is the first study to evaluate the coronal MRI view using the Proset technique for detecting the mid-zone stenosis and to define the morphologic patterns of the mid-zone stenosis using 3D MRI views (axial, sagittal, and coronal images).

\section{Materials and Methods}

This study was approved by the Institutional Review Board of Yeungnam University Hospital.

\section{Participants}

This is a retrospective exploratory study investigating the morphologic patterns of nerve root compromise at the mid-zone of the lumbar spine using MRI, especially with coronal Proset images. This study was conducted after approval of the Institutional Review Board of the first author's institution.

Of 180 patients who had compatible symptoms and/ or clinical manifestations with the lumbar pathologies and who underwent selective nerve root block for those pathologies between January 2011 and February 2013, 20 patients with mid-zone stenosis were enrolled in this study and analyzed. Mid-zone stenosis was diagnosed based on the two classifications described below.

\section{Classification}

The degree of lateral stenosis was evaluated three-dimensionally at three previously proposed zones [2]. At the axial and coronal images, the degree of compression was assessed with a modified Pfirrman classification system [7]. For the sagittal images, the degree of compression was assessed with a MRI grading system [8] summarized in Table 1.

\section{MRI settings}

MRI was performed using an Interna 1.5-T scanner (Philips Medical System, Best, The Netherlands) with a spinal array coil. Axial and sagittal T1- and turbo-T2-weighted spin-echo sequences were obtained. To evaluate morphological nerve root change in the hidden zone, 3D coronal 
Table 1. Assessment of the degree of stenosis with relation to the views of MRI

\begin{tabular}{|c|c|c|c|c|}
\hline \multirow{2}{*}{ MRI view } & \multicolumn{4}{|c|}{ Degree of stenosis } \\
\hline & Grade 0 & Grade 1 & Grade 2 & Grade 3 \\
\hline Sagittal viewa) & No & $\begin{array}{l}\text { Contact without } \\
\text { morphologic change } \\
\text { in the nerve root }\end{array}$ & $\begin{array}{l}\text { Circumferential without } \\
\text { morphologic change in } \\
\text { the nerve root }\end{array}$ & $\begin{array}{l}\text { Severe with morphologic } \\
\text { change in the nerve root }\end{array}$ \\
\hline Coronal and axial view ${ }^{\text {b) }}$ & No & Contact of nerve root & Deviation of nerve root & Compression of nerve root \\
\hline
\end{tabular}

MRI, magnetic resonance imaging; Grade 0, no; Grade 1, mild; Grade 2, moderate; Grade 3, severe.

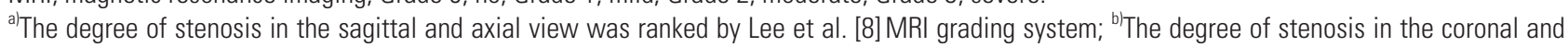
axial views were ranked by the modified Pfirrman classification [7].

fast-field echo sequences with selective water excitation were acquired using the selective excitation technique (Proset technique). The following acquisition parameters were used: section thickness, $2 \mathrm{~mm}$ with a $1 \mathrm{~mm}$ overlapping section gap; field of view, $250 \mathrm{~mm}$; 256×256 matrix; repetition time, $27.8 \mathrm{~ms}$; echo time, $13.8 \mathrm{~ms} ; 8^{\circ}$ flip angle, and two signal intensity acquisitions. Image processing consisted of direct volume rendering and segmentation, performed an Aquarius 3D workstation equipped with commercially available automated analysis 3D rendering software (TeraRecon Inc., San Mateo, CA, USA).

\section{Statistical analysis and exploration of morphological characteristics}

Conventional axial and sagittal and coronal MRI images of the stenosis were graded according to the two types of classification descibed above. The degree of stenosis was measured separately at each of the three zones (entrance, mid-, and exit-), and stenosis grade accuracy from each MRI view (axial, sagittal, and coronal views) was compared between zones. Degree of stenosis was judged by agreement between two orthopedic surgeons.

Hierarchical cluster analysis using SPSS ver. 20.0 (SPSS Inc., Chicago, IL, USA) was performed to identify morphological groups. After case classification, groups were assigned based on 3D radiologic stenosis grade at all three zones. Statistical differences among selected groups were confirmed. Briefly, the Kruskal-Wallis one-way analysis of variance for ranks (non-parametric) and the median test were both repeated until differences between groups were verified. This analysis was performed due to the study's small sample size. The cross-tabulation test was also used to confirm differences between groups. Characteristic group patterns were explored morphologically by com- paring MRI views of each group after verifying statistical differences between them.

\section{Results}

\section{Patient characteristics}

Mean patient age was 71 years (range, 59 to 85 years). Five patients were male and 15 were female. Affected nerve roots were L3 in one, L4 in nine, and L5 in 10 cases.

\section{Cluster analysis}

Stenosis grade for each zone in three dimensions are shown in Table 2. At the entrance zone, grade 0 was found in 13 of 20 cases; in the axial view, grade 1 in two cases, grade 2 in one case, and grade 3 in four cases. The median and 75th percentile grade was 0 and 1.75, respectively. Only five cases showed prominent stenosis of grade 2 or greater in the axial MRI view. At the mid-zone, prominent stenosis of grade 2 or greater was found in 14 cases in the coronal, 13 cases in the sagittal, and nine cases in the axial view. In the axial view, mid-zone stenosis was absent in six of 20 cases $(p<0.05)$. At the exit-zone, prominent stenosis of grade 2 or greater was demonstrated in 19 cases in the coronal, 12 cases in the sagittal, and 12 cases in the axial views. Severe grade 3 stenosis was most frequently seen in the coronal view ( $p=0.06$ ). All mid-zone stenosis cases in this study were associated with ipsilateral foraminal stenosis.

When these cases were classified into five groups, most of the radiological parameters were significantly different among groups after repeated statistical verification (Tables 3, 4). Statistical differences in the parameters between pairs of groups are shown in Tables 3 and 4; these parameters were used to characterize each group. Group 
Table 2. Distribution of the steosis grade in three-dimensional views of MRI at 3 zones of the lateral stenosis

\begin{tabular}{|c|c|c|c|c|c|c|c|}
\hline \multirow{2}{*}{$\begin{array}{l}\text { Degree of } \\
\text { stenosis }\end{array}$} & \multicolumn{7}{|c|}{ Radiologic parameters } \\
\hline & Entrance & Midc & Mids & Mida & Exitc & Exits & Exita \\
\hline Grade 0 & 13 & 0 & 1 & 6 & 0 & 1 & 4 \\
\hline Grade 1 & 2 & 6 & 6 & 5 & 1 & 7 & 4 \\
\hline Grade 2 & 1 & 7 & 10 & 7 & 5 & 4 & 4 \\
\hline Grade 3 & 4 & 7 & 3 & 2 & 14 & 8 & 8 \\
\hline \multicolumn{8}{|l|}{ Percentile grade } \\
\hline 25 percentile & 0 & 1 & 1 & 0 & 2 & 1 & 1 \\
\hline Median & 0 & 2 & 2 & 1 & 3 & 2 & 2 \\
\hline 75 percentile & 1.75 & 3 & 2 & 2 & 3 & 3 & 3 \\
\hline
\end{tabular}

MRI, magnetic resonance imaging; Midc, mid-zone in coronal view; Mids, mid-zone in sagittal view; Mida, mid-zone in axial view; Exitc, exit-zone in coronal view; Exits, exit-zone in sagittal view; Exita, exit-zone in axial view.

The degree of stenosis in the sagittal and axial view was ranked by Lee et al. [8] MRI grading system.

The degree of stenosis in the coronal and axial views were ranked by the modified Pfirrman classification [7].

Table 3. Distribution of the patients with relation to the median value of radiological parameters in each groups

\begin{tabular}{|c|c|c|c|c|c|c|}
\hline \multirow{2}{*}{ Radiologic parameter } & \multicolumn{5}{|c|}{ Morphologic group } & \multirow{2}{*}{$p$-value } \\
\hline & I & II & III & IV & V & \\
\hline Entrance & & & & & & $0.002^{a)}$ \\
\hline$>$ Median value & 0 & 1 & 4 & 2 & 0 & \\
\hline$\leq$ Median value & 6 & 0 & 0 & 5 & 2 & \\
\hline Midc & & & & & & 0.033 \\
\hline$>$ Median value & 0 & 1 & 2 & 2 & 2 & \\
\hline$\leq$ Median value & 6 & 0 & 2 & 5 & 0 & \\
\hline Mids & & & & & & 0.008 \\
\hline$>$ Median value & 0 & 0 & 1 & 0 & 2 & \\
\hline$\leq$ Median value & 6 & 1 & 3 & 7 & 0 & \\
\hline Mida & & & & & & 0.069 \\
\hline$>$ Median value & 2 & 0 & 1 & 6 & 0 & \\
\hline$\leq$ Median value & 4 & 1 & 3 & 1 & 2 & \\
\hline Exitc & & & & & & - \\
\hline$>$ Median value & 0 & 0 & 0 & 0 & 0 & \\
\hline$\leq$ Median value & 6 & 1 & 4 & 7 & 2 & \\
\hline Exits & & & & & & 0.010 \\
\hline$>$ Median value & 0 & 0 & 3 & 5 & 0 & \\
\hline$\leq$ Median value & 6 & 1 & 1 & 2 & 2 & \\
\hline Exita & & & & & & 0.008 \\
\hline$>$ Median value & 0 & 0 & 1 & 6 & 1 & \\
\hline$\leq$ Median value & 6 & 1 & 3 & 1 & 1 & \\
\hline
\end{tabular}

Midc, mid zone in coronal view; Mids, mid zone in sagittal view; Mida, mid zone in axial view; Exitc, exit zone in coronal view; Exits, exit zone in sagittal view; Exita, exit zone in axial view.

${ }^{\text {al) } M e a n s ~ t h e r e ~ i s ~ s t a t i s t i c a l l y ~ s i g n i f i c a n t ~ d i f f e r e n c e ~}(p<0.05)$. 
Table 4. Mean ranks with relation to morphological characteristic groups

\begin{tabular}{|c|c|c|c|c|c|c|c|}
\hline \multirow{3}{*}{ Group } & \multicolumn{7}{|c|}{ Zones } \\
\hline & \multirow{2}{*}{ Entrance } & \multicolumn{3}{|c|}{ Mid } & \multicolumn{3}{|c|}{ Exit } \\
\hline & & Coronal & Saggital & Axial & Coronal & Saggital & Axial \\
\hline Group I & 7.00 & 5.67 & 7.17 & 8.25 & 6.67 & 5.92 & 6.50 \\
\hline Group II & 16.00 & 17.00 & 4.50 & 3.50 & 13.50 & 1.00 & 2.50 \\
\hline Group III & 18.50 & 13.50 & 14.13 & 9.13 & 13.50 & 15.00 & 9.0 \\
\hline Group IV & 9.14 & 10.14 & 9.71 & 15.43 & 10.79 & 14.00 & 15.64 \\
\hline Group V & 7.00 & 17.00 & 19.00 & 6.25 & 13.50 & 7.75 & 11.50 \\
\hline$p$-value ${ }^{\mathrm{a})}$ & $0.00^{\mathrm{bl}}$ & $0.02^{b)}$ & $0.01^{\text {b) }}$ & $0.03^{b)}$ & 0.12 & $0.00^{\mathrm{bl}}$ & $0.01^{b}$ \\
\hline
\end{tabular}

${ }^{\text {a) }}$ Statistical analysis was performed by Kruskal-Wallis nonparametric test; ${ }^{b)}$ Means there is statistically significant difference $(p<0.05)$.

composition was as follows: six cases in group I, one case in group II, four cases in group III, seven cases in group $\mathrm{IV}$, and two cases in group V.

At the entrance zone, stenosis mean rank was 16 and 18.5 in group II and group III, respectively $(p<0.05)$. All four cases in group III showed severe grade 3 stenosis, the most prominent severe stenosis $(p<0.05)$ (Table 4$)$.

At the mid-zone, stenosis mean rank in the coronal plane was 17 for groups II and V; all cases in these groups demonstrated grade 3 stenosis. In groups III and IV, stenosis mean rank in the coronal plane was 13.5 and 10.1, respectively. Two cases in each group were grade 3 and the other seven cases exhibited grade 2 or lower stenosis. These findings suggested that groups II and V exhibited severe up-down stenosis at the mid-zone, whereas groups III and IV exhibited moderate to severe up-down stenosis. In the sagittal plane, group V mean rank was 19, and two cases in this group exhibited grade 3 stenosis $(p<0.05)$. Group II mean rank was 4.5 , and front-back stenosis was grade 1 . In the axial view, mean group IV rank was 15.4; moreover, six of seven cases showed grade 2 or greater nerve root compression. In the other groups, the extent of nerve root compression was not as great as in group IV $(p<0.05)$.

At the exit zone, the 25th percentile for stenosis in the coronal plane was grade 2, and 19 of 20 cases exhibited moderate to severe grade 2 or greater stenosis. Thus, stenosis in the coronal plane at the exit-zone was not significantly different among the five groups. In the sagittal plane, mean ranks were 15.0 and 14.0 for groups III and IV, respectively; furthermore, all eight cases of stenosis in these groups were severe (grade 3$)(p<0.05)$. In the axial view, average ranks were 15.6 and 11.5 for groups IV and
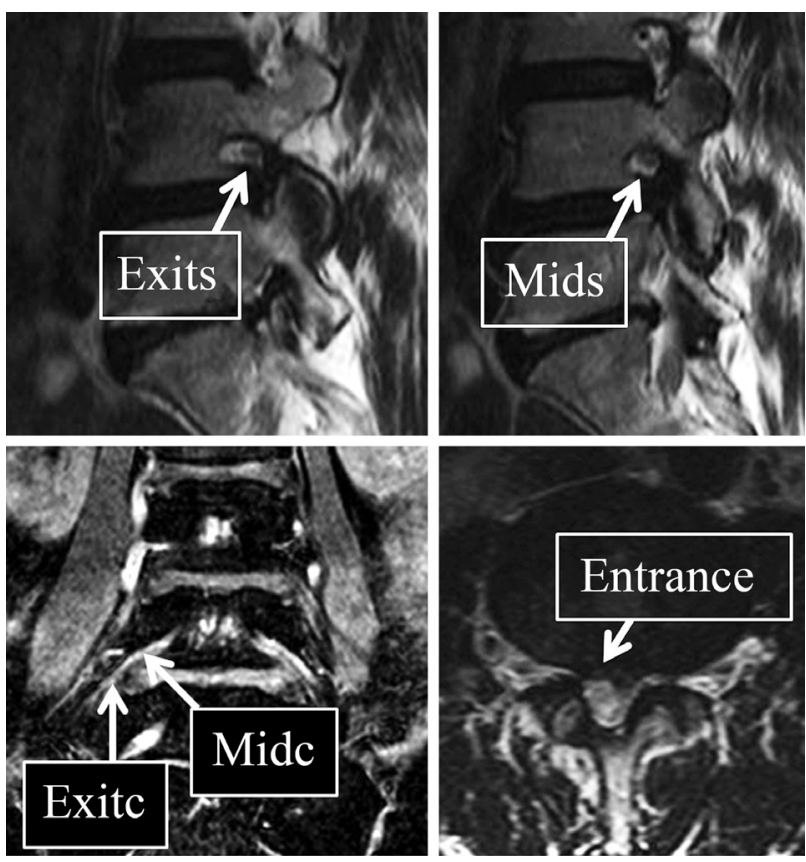

Fig. 1. Group I data. Proset magnetic resonance imaging coronal views reveal mild compression of the right $L 4$ nerve root along the lateral nerve root canal in mid- and exit-zones. Stenosis degree at the midzone is grade 1 , and that at the exit zone is grade 2 . In the sagittal view, the exi-zone is grade 1. midc, mid zone in coronal view; mids, mid zone in sagittal view; exitc, exit zone in coronal view; exits, exit zone in sagittal view.

$\mathrm{V}$, respectively. Six of eight cases in group IV were grade 3 $(p<0.05)$.

\section{Morphological characteristics}

Group I was characterized by a mild degree of stenosis in all directions and in all three zones (Fig. 1). Of all five 

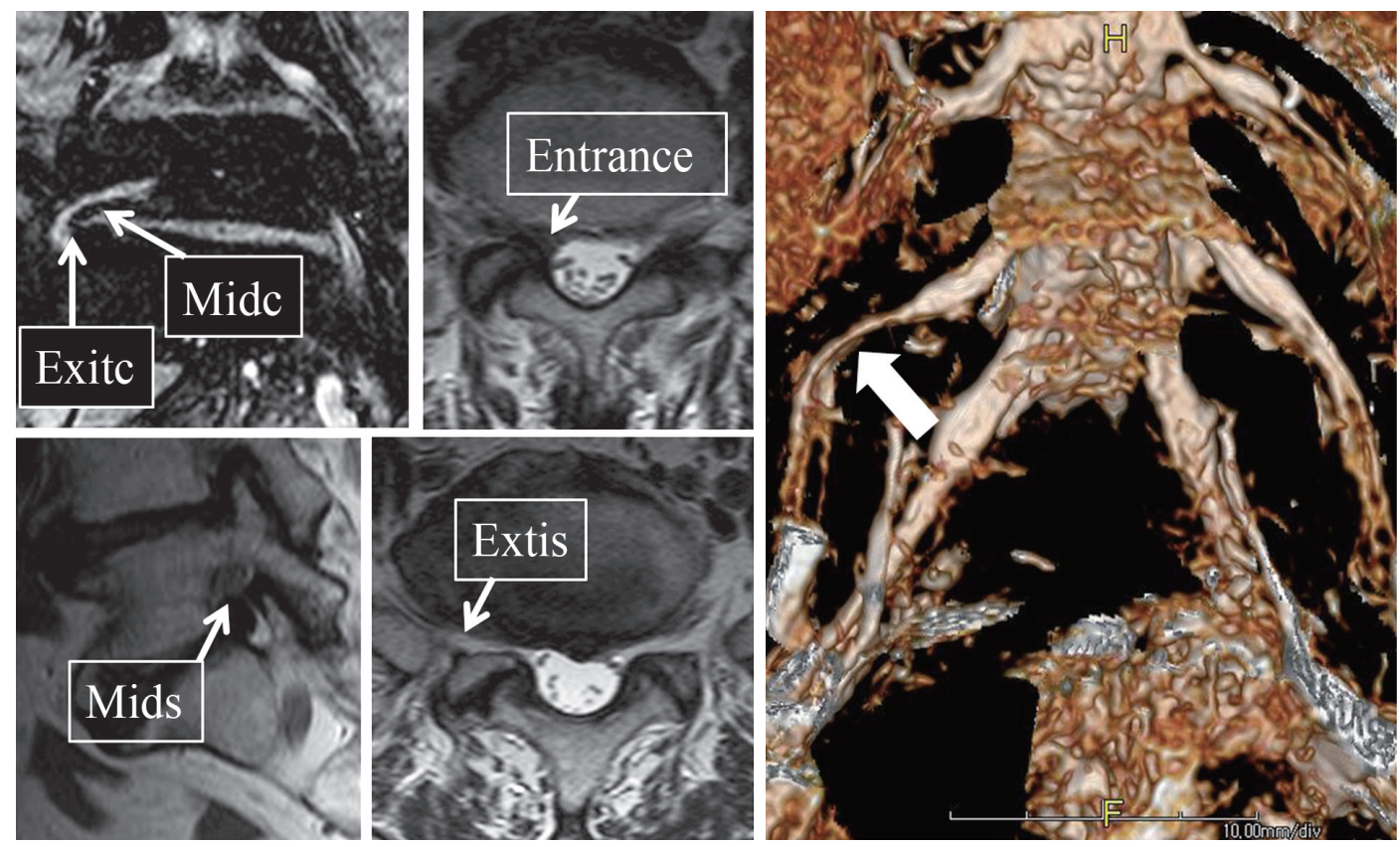

Fig. 2. Group II data. Proset magnetic resonance imaging (MRI) coronal views reveal severe grade 3 up-down stenosis at the right L5 nerve root along the lateral nerve root canal in the mid- and exit-zones. Sagittal stenosis is grade 1 or 0 at the mid- and exit-zones, respectively, and is not remarkable even in the axial view. At its entrance zone, the nerve root is displaced anteriorly and compressed. Severe compression (white arrow) of the right L5 nerve root in up-down direction is well visualized in the three-dimensional Proset MRI. midc, mid zone in coronal view; mids, mid zone in sagittal view; exitc, exit zone in coronal view; exits, exit zone in sagittal view.

groups, this group exhibited the mildest degree of compression (grade 1 or zero) along the nerve root canal. Group II showed only one case of severe up-down stenosis at the mid- or exit-zones in the coronal plane and no front-back stenosis in the sagittal plane. Fig. 2 shows the flattened nerve root in this case, which was due to severe up-down stenosis at the mid- and exit-zones. This flattened nerve root was also associated with hypertrophied osteophytes at the lower end plate of the L5 vertebral body and disc protrusion in the up-ward direction, with disc space narrowing. However, prominent front-back stenosis was not observed. Groups III and IV were characterized by moderate stenosis at the mid-zone associated with severe stenosis at the exit zone (Figs. 3, 4). Group III demonstrated severe grade 3 entrance zone stenosis in all four cases, but this type of stenosis was not observed in group IV $(p<0.05)$. Rather, group IV was characterized by moderate to severe stenosis along the entire nerve root canal, extending from the entrance zone to the exit-zone. Degree of stenosis in the axial view at the mid- and exit-zone was more severe in group IV than in group III $(p<0.05)$ (Fig. 4). Group $V$ was characterized by severe circumferential stenosis at the mid-zone, in addition to severe up-down

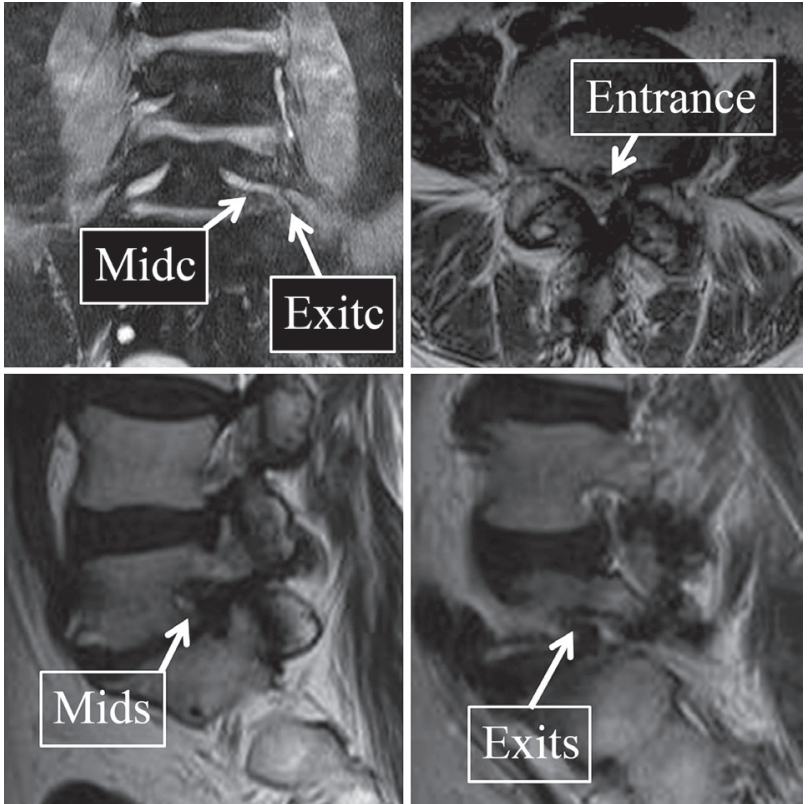

Fig. 3. Group III data. Proset magnetic resonance imaging (MRI) coronal and conventional sagittal view reveal moderate grade 2 circumferential stenosis at the mid-zone and severe circumferential stenosis at the exit-zone of the left $L 4$ nerve root. The axial view showed severe grade 3 central and entrance zone stenosis. midc, mid zone in coronal view; mids, mid zone in sagittal view; exitc, exit zone in coronal view; exits, exit zone in sagittal view. 


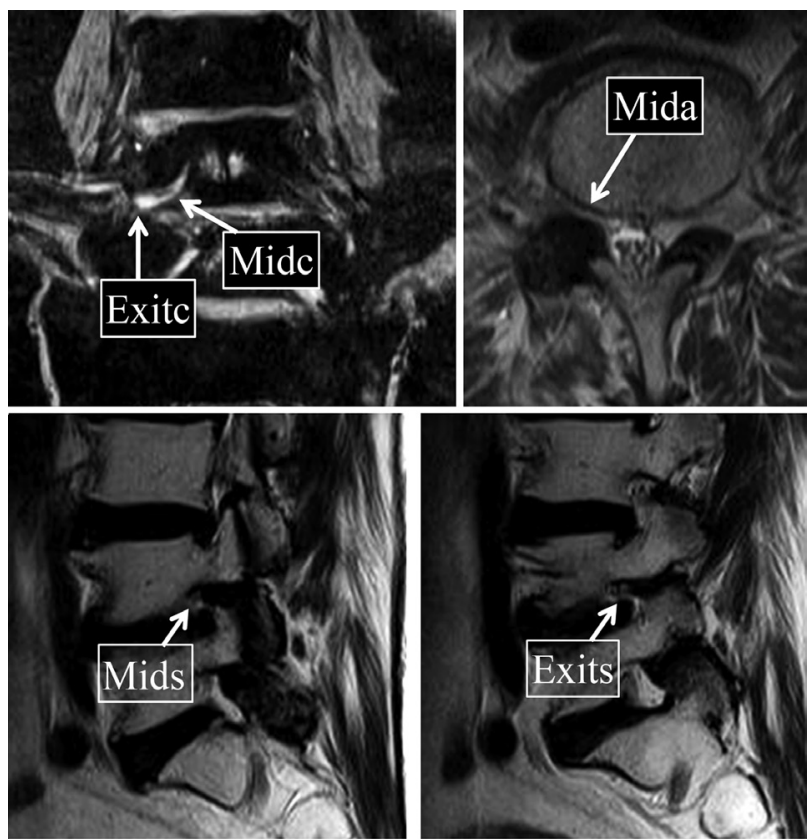

Fig. 4. Group IV data. Proset magnetic resonance imaging coronal and conventional sagittal view reveal moderate to severe up-down and circumferential grade 2 or 3 stenosis at the right $L 4$ nerve root along the mid- and exit-zones. The axial view shows severe grade 3 stenosis at the mid- and exit-zone of the right $L 4$ nerve root canal. midc, mid zone in coronal view; mids, mid zone in sagittal view; mida, mid zone in axial view; exitc, exit zone in coronal view; exits, exit zone in sagittal view.

stenosis at the exit-zone, without a remarkable degree of entrance zonal stenosis (Fig. 5). Both coronal views of a Proset MRI image and conventional sagittal views revealed severe circumferential grade 3 stenosis at the midzone, with no evidence of stenosis in the axial view. At the exit-zone, severe up-down grade 3 stenosis was noted in the coronal view, whereas moderate grade 2 stenosis was observed in the sagittal view. No evidence for stenosis at the entrance zone was observed. The mid- and exit-zones were most severely compromised by a hypertrophied joint capsule and a hypertrophied lower end plate with upward intervertebral disc protrusion.

\section{Discussion}

Routine surgical procedures for stenotic lesions in the lumbar spine can be extremely difficult in some cases due to anatomical peculiarity that obscure mid-zone stenosis in preoperative diagnostic modalities. Furthermore, incomplete decompression due to improper preoperative diagnosis and surgical planning is one of the most
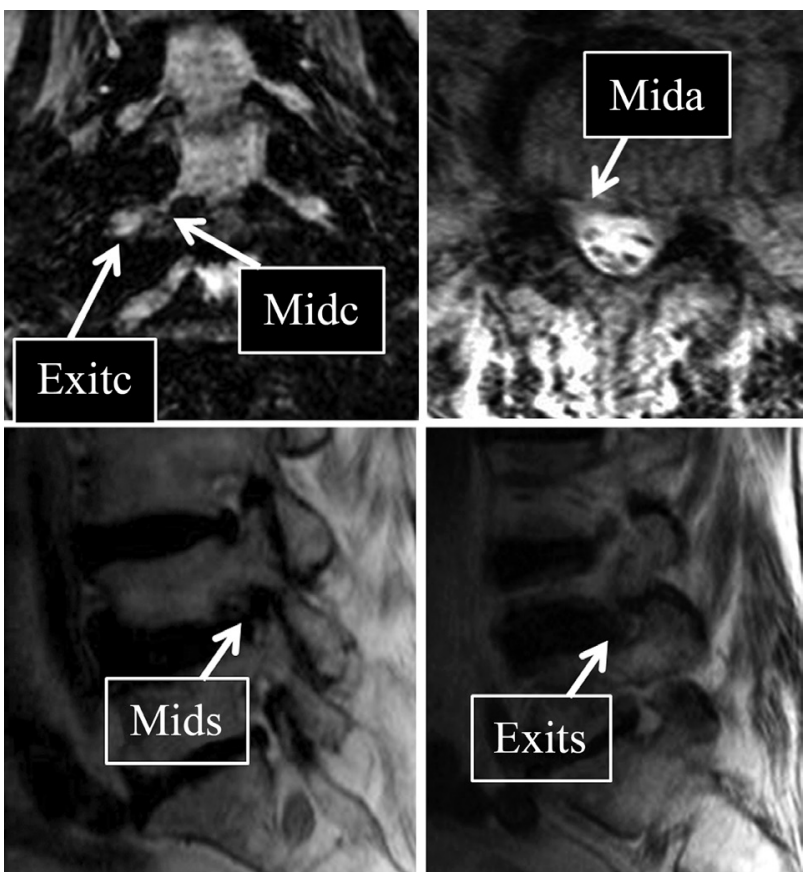

Fig. 5. Group V data. Proset magnetic resonance imaging coronal and conventional sagittal views reveal severe circumferential grade 3 stenosis at the right $L 4$ nerve root, with no evidence of stenosis in the axial view. At the exit zone, severe up-down stenosis is noted in the coronal (grade 3) and sagittal view (grade 2). There is no evidence of stenosis at the entrance zone of the right $L 4$ nerve root. midc, mid zone in coronal view; mids, mid zone in sagittal view; mida, mid zone in axial view; exitc, exit zone in coronal view; exits, exit zone in sagittal view.

important causes of failed back surgery syndrome [9-12]. In particular, nerve root compromise or stenotic lesions at the mid-zone has been shown to be a typical example where lesions can easily be missed during preoperative evaluations. Macnab [1] aimed to detect compression lesions around the pedicle using conventional myelogram. However, the strategy proved unsuccessful. Thus, this region came to be known as the hidden zone. The development of new diagnostic modalities, especially MRI, may aid in hidden zone detection [13-15]. However, difficulty detecting mid-zone lesion with conventional radiologic examinations even with CT and MRI was also stressed in that study.

Stenotic lesion at the mid-zone, which is located inferomedial to the pedicle, are not always detected in conventional axial or sagittal MRI scans. Conventional axial and sagittal MRI views enable visualization of front-back and up-down stenosis around the foramen. However, these views are not adequate for fully visualizing the entire compressed nerve root in the compromised canal. The present study demonstrated that the coronal view enabled more 
precise visualization of the nerve root than any other view at the mid-zone. In particular, the Proset coronal view facilitated more accurate detection of the nerve root signal intensity and its related stenotic lesion; this improved accuracy is likely achieved by Proset's prominent water enhancement feature. This enhancement could aid in the detection of nerve root compression at the mid-zone, a hypothesis that is supported by this study's findings, which suggest that mid-zone stenosis exhibits variable $3 \mathrm{D}$ pathological changes, similar to stenosis in other zones. Mid-zone stenosis can be hidden in conventional MRI views. A single case in group II had up-down stenosis detected only in the coronal view, with no evidence to indicate its presence in any of the conventional views. These findings suggest that $3 \mathrm{D}$ analysis using the coronal as well as conventional axial and sagittal images is important for visualizing pathological changes around the pedicle.

To our knowledge, no study has yet focused on the morphological differences of stenotic lesions in the lumbar spine, especially mid-zone stenosis. In this study, individual morphological differences in mid-zone stenosis characteristics were investigated statistically using cluster analysis. As well, group morphological characteristics were carefully verified. Specific patterns of mid-zone stenosis were identified, including mild degree of stenosis, in all directions and all three zones (group I); up-down stenosis alone without remarkable front-back stenosis (group II); moderate to severe stenosis along the entire nerve root canal, extending from the entrance to exit-zones (group III and IV, according to the degree of severity in axial image); and severe circumferential stenosis along the midand exit-zones, without remarkable entrance zone stenosis (group V).

Lee et al. [2] pointed out that most mid-zone stenosis is associated with stenosis in other zones, especially foraminal stenosis. This finding is consistent with those of the present study. To avoid incomplete decompression and iatrogenic instability, decompression of the entrance and exit-zones followed by complete removal of all mid-zone hypertrophied osteophytes is recommended. However, this procedure is technically demanding and fails to take into account individual patterns of stenosis and the severity of each stenosis instance when selecting the most appropriate surgical approach. In cases with mild to moderate stenosis localized to a limited area, such as in groups I-IV (but not in group V), this recommendation could be successful [2]. Several recent modifications to the poste- rior surgical techniques, including the muscle-preserving inter-laminar approach and the contra-lateral approach, may be helpful in maintaining the postoperative stability of the affected segment [16-18]. However, it is very difficult to obtain stability after surgery, especially in cases with severe circumferential stenosis in group $\mathrm{V}$ or severe stenosis along the entire nerve root canal as seen in group III. Most mid-zone stenosis is up-down stenosis and is usually associated with disc space narrowing; moreover, this type of stenosis usually occurs with foraminal stenosis. Thus, decompression of mid-zone stenosis cannot be performed without disturbing the stability of the facet joint. In such cases, transforaminal or posterior lumbar interbody fusion may be preferable as my personal suggestion, for reasons of neurological and mechanical safety.

This study had some limitations. One was the relatively small sample size that only included mid-zone stenosis cases treated with selective nerve root injection. However, since few reference studies are available, the characteristics of mid-zone stenosis in foraminal or entrance zone stenosis has been poorly understood. The measurement of the radiologic images was done by a single orthopaedic spine surgeon in the current study, which was another limitation because the measurements were performed by several raters with analyzing the inter- and intra-rater reliability. We performed this study without considering patients' clinical symptoms or manifestations, but just evaluated radiologic images for detecting the hidden zone. In addition, this study was the first to thoroughly evaluate coronal MRI utility for diagnosing mid-zone stenosis and showed that coronal Proset MRI enabled hidden midzone stenotic lesion detection that was not possible with conventional axial and sagittal MRI. This study also outlined optimal surgical approaches for different patterns of stenosis and aimed to more precisely evaluate coronal Proset MRI utility. However, these approaches were not tested in this study. Thus, a further large-scale study focused on mid-zone stenosis, including an exploration of optimal surgical methods for each stenosis pattern, is needed.

\section{Conclusions}

The hidden zone in patients with mid-zone stenosis is still valid; this zone represents the area difficult to image with conventional axial and sagittal MRI, due to individual anatomical peculiarity. The mid-zone is just inferomedial 
to the pedicle and pars interarticularis and exhibits variable morphological pattenrs from individual to individual, as shown in our classification. Based on the current study, the coronal MRI with Proset technique is needed to better define the mid-zone stenotic lesions along the entire nerve root.

\section{Conflict of Interest}

No potential conflict of interest relevant to this article was reported.

\section{References}

1. Macnab I. Negative disc exploration: an analysis of the causes of nerve-root involvement in sixty-eight patients. J Bone Joint Surg Am 1971;53:891-903.

2. Lee CK, Rauschning W, Glenn W. Lateral lumbar spinal canal stenosis: classification, pathologic anatomy and surgical decompression. Spine (Phila Pa 1976) 1988;13:313-20.

3. Byun WM, Ahn SH, Ahn MW. Value of 3D MR lumbosacral radiculography in the diagnosis of symptomatic chemical radiculitis. AJNR Am J Neuroradiol 2012;33:529-34.

4. Byun WM, Jang HW, Kim SW. Three-dimensional magnetic resonance rendering imaging of lumbosacral radiculography in the diagnosis of symptomatic extraforaminal disc herniation with or without foraminal extension. Spine (Phila Pa 1976) 2012;37: 840-4.

5. Kim MH, Suh KJ, Lee JY, Min SH, Yoo HY. Usefulness of coronal MR image in diagnosis of foraminal and extraforaminal disc herniation. J Korean Soc Spine Surg 2008;15:165-73.

6. Ahn MW, Kim US, Kim GB. Usefulness of the Proset MRI for diagnosis of the extraforaminal disc herniation. J Korean Soc Spine Surg 2013;20:28-33.

7. Pfirrmann CW, Dora C, Schmid MR, Zanetti M, Hodler J, Boos N. MR image-based grading of lumbar nerve root compromise due to disk herniation: reliability study with surgical correlation. Radiology 2004;230:583-8.

8. Lee S, Lee JW, Yeom JS, et al. A practical MRI grading system for lumbar foraminal stenosis. AJR Am J
Roentgenol 2010;194:1095-8.

9. Lee GY, Lee JW, Choi HS, Oh KJ, Kang HS. A new grading system of lumbar central canal stenosis on MRI: an easy and reliable method. Skeletal Radiol 2011;40:1033-9.

10. Guyer RD, Patterson M, Ohnmeiss DD. Failed back surgery syndrome: diagnostic evaluation. J Am Acad Orthop Surg 2006;14:534-43.

11. Fritsch EW, Heisel J, Rupp S. The failed back surgery syndrome: reasons, intraoperative findings, and longterm results: a report of 182 operative treatments. Spine (Phila Pa 1976) 1996;21:626-33.

12. Waguespack A, Schofferman J, Slosar P, Reynolds J. Etiology of long-term failures of lumbar spine surgery. Pain Med 2002;3:18-22.

13. Nakao S, Yoshida M, Yamada H, Hashizume H. A new 3-dimensional computed tomography imaging method to diagnose extraforaminal stenosis at the lumbosacral junction. J Spinal Disord Tech 2010;23: e47-52.

14. Alicioglu B, Yilmaz B, Bulakbasi N, et al. Magnetic resonance imaging predictors of surgical outcome in degenerative lumbar spinal stenosis. Jpn J Radiol 2012;30:811-8.

15. Park HJ, Kim SS, Lee SY, et al. Clinical correlation of a new MR imaging method for assessing lumbar foraminal stenosis. AJNR Am J Neuroradiol 2012;33: 818-22.

16. Yoshimoto M, Miyakawa T, Takebayashi T, et al. Microendoscopy-assisted muscle-preserving interlaminar decompression for lumbar spinal stenosis: clinical results of consecutive 105 cases with more than 3-year follow-up. Spine (Phila Pa 1976) 2014;39: E318-25.

17. Hatta Y, Shiraishi T, Sakamoto A, et al. Muscle-preserving interlaminar decompression for the lumbar spine: a minimally invasive new procedure for lumbar spinal canal stenosis. Spine (Phila Pa 1976) 2009; 34:E276-80.

18. Bernucci C, Giovanelli M. Translaminar microsurgical approach for lumbar herniated nucleus pulposus (HNP) in the "hidden zone": clinical and radiologic results in a series of 24 patients. Spine (Phila Pa 1976) 2007;32:281-4. 